\title{
Impact of protected mealtimes on mealtime environment and nutrient intake in hospital patients
}

\author{
A. Connolly ${ }^{1}, \mathrm{~K}$. Whelan ${ }^{1}$ and M. Hickson ${ }^{2}$ \\ ${ }^{1}$ Nutritional Sciences Division, King's College London, London, UK and ${ }^{2}$ Dietetic Research Group, Imperial College \\ Healthcare NHS Trust, London, UK
}

\begin{abstract}
Malnutrition is reported in 13-40\% of hospital patients and is associated with poor clinical outcomes and high health-care costs ${ }^{(1)}$. Despite the lack of high-quality evidence, recent government strategies have focused on the provision of adequate nutritional care to alleviate malnutrition. Protected mealtimes (PM) are one of the Council of Europe's 10 key characteristics of good nutritional care in hospitals ${ }^{(2)}$. The aim of this observational 'before $v$. after' audit was to compare mealtime environment and nutrient intake pre- and post-PM implementation in an acute hospital trust.

Data were collected at the baseline (2008) and following PM implementation approximately one year later (2009). A mealtime questionnaire relating to the PM objectives was completed by two observers (dietetic and nursing) for a sample of patients on all wards. Patients screened as high risk for malnutrition and not receiving artificial nutrition were considered for participation of a weighed food intake evaluation. Characteristics influencing food intake were recorded for each patient (gender, disease category, etc.) and were similar in the pre-PM and PM analysis. The researcher weighed wastage one lunch time meal and nutrient intake was estimated based upon the average serving size. Categorical data were analysed using the Chi-squared test and continuous data were analysed using the MannWhitney $U$-test.

Questionnaires were analysed for 253 and 237 individual patients on 40 and 34 wards pre-PM and post-PM, respectively. Nineteen PM objectives were measured and significant improvements were shown in only three: patients on food and/or fluid charts (32.1\% v. $42.7 \%$, $P=0.021)$, opportunity to clean hands prior to eating $(30.0 \% v .40 .1 \%, P=0.029)$ and clean and clear tables $(54.3 \% v .63 .9 \%$, $P=0.039)$. There was no significant difference in the proportion of patients experiencing mealtime interruptions $(68.3 \% v .74 .8 \%$, $P=0.143$ ). Thirty-nine at pre-PM and 60 patients at post PM had their meal waste measured and group characteristics were comparable. There was no significant difference in energy or protein served to each group. There was no significant difference in energy intake $(1087.84 \mathrm{~kJ}(260 \mathrm{kcal})$ v. $836.8 \mathrm{~kJ}(200 \mathrm{kcal}), P=0.246)$; however, the PM group consumed significantly less protein $(7.5 \mathrm{~g} v .14 \mathrm{~g}$, $P=0.039)$.

This study showed that only small improvements were made in the PM environment on the wards. This improvement did not include the important criterion of reducing mealtime interruptions. The observed improvements in cleanliness of environment and nutritional monitoring were not enough to increase nutritional intake. The reason for a lower protein intake is unclear. These findings highlight the need for an evidence-based PM implementation method that produces measurable improvements in all PM objectives and thus enabling a reliable measure of the impact of PM on nutritional and clinical outcomes.
\end{abstract}

1. Elia, M, Stratton R, Russell C et al. (2005) The cost of disease-related malnutrition in the UK and economic considerations for the use of oral nutritional supplements [online]. Available: http://www.bapen.org.uk/res_reports.html (accessed 25 May 2010).

2. Council of Europe Alliance. (2003) Council of Europe Resolution Food and Nutritional Care in Hospital [online]. Available: http://www.bapen.org.uk/ pdfs/coe_leaflet.pdf (accessed 1 May 2010). 